\title{
Percepciones de servicios y productos de la librería de la UNAH en Tegucigalpa, 2016.
}

\author{
Reina Lovo ${ }^{1}$ \\ Fernando Flores ${ }^{2}$ \\ Lucy Gómez ${ }^{3}$
}

\section{RESUMEN}

El propósito de este estudio es conocer la percepción de la comunidad universitaria (docentes, personal administrativo y de servicio) de la UNAH Tegucigalpa sobre la calidad de los productos y servicios que ofrece la Librería universitaria, durante el segundo periodo del 2016, de igual manera, se ausculta sobre las condiciones en las que opera actualmente la Librería Universitaria y de qué manera esto influye en la satisfacción de los clientes. La necesidad de información surge de la Secretaria de Desarrollo Institucional (SEDI), que solicita una investigación para conocer la situación actual de los servicios brindados en la Librería Universitaria, con la finalidad de tomar decisiones y diseñar estrategias para la mejora continua. Esta investigación es de carácter mixto con enfoque cualitativo y cuantitativo con un diseño concluyente, descriptivo transversal simple donde se obtuvo información de estudiantes, docentes, personal administrativo y de servicios (total 775 encuestados) y de los empleados (10 entrevistas) que laboran en la Librería Universitaria. La principal conclusión es que el nivel de satisfacción con respecto a los productos y servicios que se oferta en la librería es de la categoría de satisfechos. Entre las recomendaciones están que se ofrezcan precios más bajos en los libros y productos, mejorar la atención al cliente por parte de los colaboradores, que se amplíe el área de la librería, que exista más variedad de textos, así como de autores de los mismos y ofrecer mayor variedad de literatura.

Palabras clave: productos, servicios, satisfacción, calidad, percepción, comunidad universitaria.

\footnotetext{
${ }_{1}^{1}$ Profesora del Departamento de Mercadotecnia, Facultad de Ciencias Económicas, Administrativas y Contables, UNAH: reina.lovo@unah.edu.hn

2 Profesor del Departamento de Mercadotecnia, Facultad de Ciencias Económicas, Administrativas y Contables, UNAH: fernando.flores@unah.edu.hn

${ }^{3}$ Profesora del Departamento de Mercadotecnia, Facultad de Ciencias Económicas, Administrativas y Contables, UNAH: lucy.gomez@unah.edu.hn
} 


\section{ABSTRACT}

The purpose of this study is to analyze the perception of the university community (teachers administrative and service staff) of the UNAH Tegucigalpa on the quality of products and services offered by the University Library during the second period of 2016, we analyzed the conditions in which the University Library currently operates and how this influences customer satisfaction. The need for information arises from the Secretariat for Institutional Development (SEDI), from which an investigation was requested to help them to know the current situation of the services offered in the university library with the purpose of making decisions and design strategies for improvement keep going. This research is mixed with a qualitative and quantitative approach, with a conclusive and descriptive simple transversal design, where information was obtained from students, teachers, administrative staff (775 respondents) and collaborators (10 interviews) working at the university library.

Among the most important conclusions are that the level of satisfaction with respect to the products and services offered in the bookstore is "satisfied". Recommending that lower prices must be offered in books and products, improving customer service by the collaborators, expanding the area of the bookstore, providing more variety of texts as well as authors and offering more variety of literature.

Keywords: Products, services, satisfaction, quality, perception, university community. 


\section{INTRODUCCIÓN}

La Secretaria de Desarrollo Institucional de la UNAH (SEDI), realiza investigaciones que permitan obtener información relevante para poder diseñar estrategias que conllevan a la mejora de cada una de las unidades. En esta ocasión, se solicitó una investigación que le ayudará a conocer la situación actual de los servicios brindados en la librería, su desempeño y la percepción de la comunidad universitaria sobre la calidad de los servicios y productos que se ofertaron durante II periodo del 2016.

El estudio tiene como propósito proveer insumos importantes a las autoridades universitarias quienes, en la búsqueda de la mejora continua, se han propuesto contribuir al logro de las metas institucionales y al desarrollo del país, llevando a la universidad a contribuir en la búsqueda de una educación globalizada, con altos estándares de servicio y mejorar posiciones en el ranking de universidades.

\section{CONTENIDO}

Para cualquier institución que decide ofertar sus productos y servicios, es necesario conocer como lo está haciendo y que debería mejorar. El ofertar no solo se trata vender un objeto físico, es saber ofrecer los beneficios que lleven al cumplimiento de las expectativas del cliente durante la adquisición de un producto.

Desde el punto de vista de la mercadotecnia tanto los productos tangibles como los intangibles se conciben como proporcionadores de la satisfacción, necesidades y deseos de los consumidores, por lo tanto, las organizaciones que brindan servicios deberán poner estricta atención a estos, aunque no se dediquen a elaborar productos directamente.

Para comprender mejor esta idea, es importante señalar la diferencia entre producto y servicio. Según Kotler, 2012 define:

Producto como cualquier cosa que se puede ofrecer a un mercado para su atención, adquisición, uso o consumo y que podría satisfacer un deseo o una necesidad y los servicios son una forma de producto que consiste en actividades, beneficios 0 satisfacciones que se ofrecen en venta y que son esencialmente intangibles y no tienen como resultado la propiedad de algo. (pp.224) 


\section{Percepción}

Para (Kerin, 2014) percepción es el proceso por el cual el individuo selecciona, organiza e interpreta la información para formarse una imagen significativa del mundo, a su vez clasifica la percepción en dos grandes ramas, la percepción selectiva y la percepción subliminal.

La percepción selectiva es aquella donde el consumidor común y corriente opera en un entorno complejo de modo que el cerebro humano, este a su vez filtra e interpreta la exposición, comprensión y retención de los mensajes y en la percepción subliminal significa que la persona ve o escucha mensajes sin estar consciente de ello.

\section{Satisfacción}

Philip Kotler, establece que la mercadotecnia es una actividad humana que cuya finalidad consiste en satisfacer necesidades y deseos del ser humano mediante el proceso de intercambio.

Así mismo, (Kotler \& Armstrong, Fundamentos de Marketing, 2012) describen la satisfacción como el grado en que el desempeño percibido de un producto concuerda con las expectativas del comprador. Si el desempeño del producto no alcanza las expectativas del comprador, quedará insatisfecho, por lo tanto, la satisfacción depende del desempeño que se perciba de un producto en cuanto a la entrega de valor.

El cliente, percibe el valor cuando evalúa las diferencias entre todos los beneficios y costos de una oferta de marketing con relación a la oferta de la competencia.

\section{Servicio}

Según (Kotler, Fundamentos de Marketing, 2014) las características y naturaleza de un servicio van enfundadas en cuatro aspectos:

- Intangibilidad de los servicios: Implica que los servicios no se pueden ver, degustar, tocar ni oír.

- Inseparabilidad de servicios: implica que los servicios no se pueden separar de los proveedores, sean estas personas o máquinas.

- Variabilidad de los servicios: Implica que la calidad de los servicios depende de quién lo presta, además de cuándo, dónde y cómo se presentan. 
- Caducidad de los servicios: Implica que los servicios no se pueden almacenar para venderse o usarse posteriormente.

\section{Producto}

Un producto en Mercadotecnia es hablar de todo aquello que se puede comercializar o vender, es por ello que Kotler define producto como cualquier cosa que se puede ofrecer a un mercado para su atención, adquisición, uso o consumo y que podría satisfacer un deseo o una necesidad y pueden ser tangibles e intangibles (Kotler, Fundamentos de Marketing, 2014).

Lo importante en relación a los planteamientos antes descritos, es que aunque la satisfacción del cliente es un parámetro que parece sencillo de determinar - por depender básicamente de dos aspectos (expectativas y percepciones) - es bastante complejo, ya que involucra la manera como las personas perciben la calidad, lo cual lleva inmersos un sin fin de aspectos psicológicos que varían de cliente en cliente, incluso cuando se trata de prestar un mismo servicio.

\section{Definición de oferta, según expertos en Mercadotecnia y Economía}

La oferta se refiere a "las cantidades de un producto que los productores están dispuestos a producir a los posibles precios del mercado". Complementando ésta definición, ambos autores indican que la ley de la oferta "Son las cantidades de una mercancía que los productores están dispuestos a poner en el mercado, las cuales, tienden a variar en relación directa con el movimiento". Laura Fisher y Jorge Espejo, autores del libro "Mercadotecnia" (Fisher \& Espejo, 2010),

\section{FUNDAMENTACIÓN METODOLÓGICA}

Esta investigación es de carácter mixto con enfoque cualitativo y cuantitativo con un diseño concluyente, descriptivo transversal simple donde se obtuvo información de estudiantes, docentes, personal y de servicio (total 775 encuestados), de los colaboradores (10 entrevistas) que laboran en la librería universitaria. Previamente se realizó la investigación exploratoria, a través de la observación y la técnica de cliente oculto o simulado (Mistery Shopper). La muestra se calculó con base al dato poblacional de docentes, estudiantes, personal administrativo y de servicio según estadísticas en el anuario de la UNAH, del II período del 2016. La técnica de muestreo es no 
probabilística, por cuotas distribuidas entre las carreras proporcionalmente a la matricula (estudiantes) y cantidad de empleados por facultad. No se cuenta con antecedentes de la existencia de un estudio previo que esté registrado en alguna unidad de la UNAH.

\section{TÉCNICAS DE RECOLECCIÓN DE DATOS}

Los instrumentos de medición que se seleccionaron son:

- La entrevista a experto (aplicada al ex director de la librería).

- La check list (para el mistery shopper).

- El cuestionario (aplicado a la comunidad universitaria y colaboradores).

\section{PROCESAMIENTO Y ANÁLISIS DE LOS DATOS}

Se realizó el proceso de edición de los cuestionarios y después se procedió a la transcripción de los cuestionarios, se elaboró una plantilla en el programa SPSS para la tabulación. Para el análisis de los datos, se realizó un análisis univariado y multivariado y se presentan cruces de variables para realizar un análisis de mayor profundidad. Las tablas y gráficas se editaron en el programa Excel.

\section{RESULTADOS}

Gráfico 1. Orden de los productos

\begin{tabular}{r|c|} 
Satisfecho & 59.6 \\
Muy Satisfecho & 14.9 \\
Ins atisfecho & 13.1 \\
No A.plica & 10.1 \\
\hline Muy Insatisfecho & 2.2
\end{tabular}

Fuente: Elaboración propia 


\section{Gráfico 2. Disponibilidad de productos-cafetería}

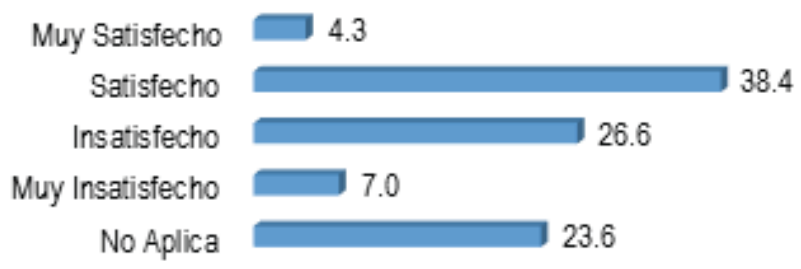

Fuente: Elaboración propia

Las personas dentro de la comunidad universitaria, según el gráfico 1, muestran un $59.6 \%$ de satisfacción seguido de un $14.9 \%$ que están muy satisfechos respecto al orden en que los productos están colocados, sin embargo, un 13.1\% manifestó estar insatisfecho. En relación a la disponibilidad de productos que se ofrecen en la cafetería, se puede observar en el gráfico 2 , que el $38.4 \%$ de las personas muestran satisfacción, sin embargo, un 26.6 \% muestra insatisfacción.

Con respecto a la variedad de los productos que se encuentran disponibles en la Librería Universitaria, el $43.6 \%$ de las personas están satisfechas y el $27.0 \%$ está insatisfecho según el gráfico 3 , son porcentajes que develan poca conformidad en esta variable fundamental de los servicios ofertados en la Librería universitaria.

\section{Gráfico 3. La variedad de los productos responde a las necesidades del usuario}

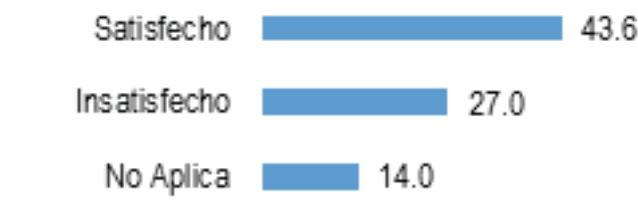

Mury Insatisfecho

8.1

Muy Satisfecho

7.3

Fuente: Elaboración propia

La calidad de los otros productos (uniformes. útiles y artículos promocionales) que se ofrecen en la librería, el gráfico 4 revela un $47.8 \%$ de satisfacción, aunque la variable precio muestra porcentajes de poca satisfacción ya que solo el $35.3 \%$ está satisfecho y un $26.8 \%$ muestra insatisfacción según se observa en el gráfico 5 . 


\section{Gráfico 4. Calidad de los productos}

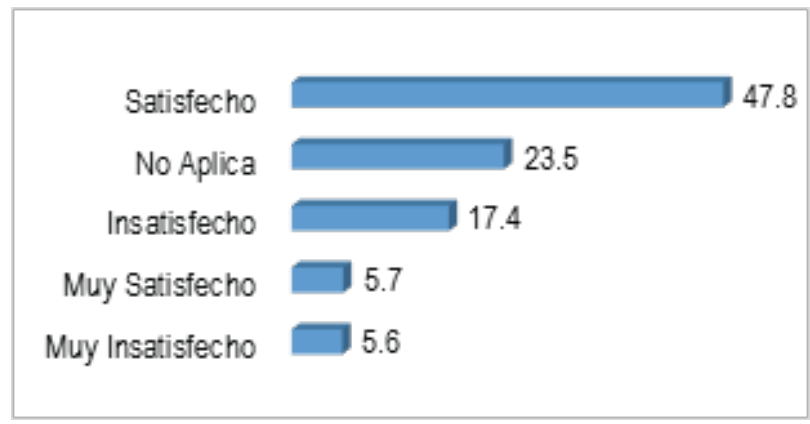

Fuente: Elaboración propia

\section{Gráfico 5. Precios de los productos}

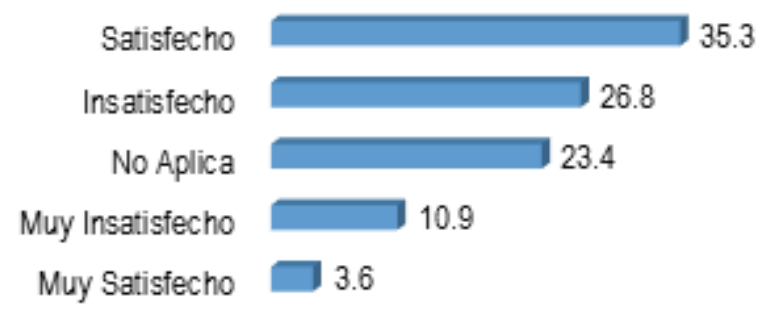

Fuente: Elaboración propia

El siguiente gráfico muestra la opinión sobre los productos más demandados que son los uniformes (40\%) y la ropa de vestir con serigrafía de la UNAH $(30 \%)$.

\section{Gráfico 6. Productos de mayor preferencia}

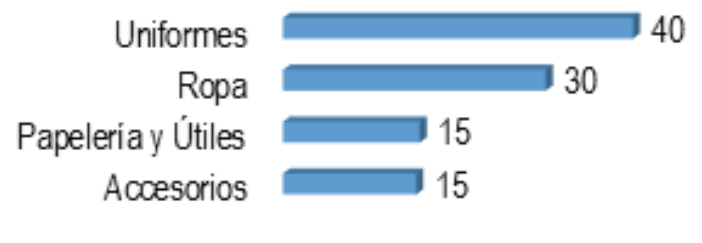

Fuente: Elaboración propia 
El gráfico 7 muestra el desempeño de los empleados que es evaluado en general como satisfactorio ya que las personas consideran son amables (45.7\%), y en el gráfico 8 , se menciona que los empleados muestran disposición para ayudar a los clientes (42.6\%), sin embargo, es importante denotar que los resultados igualmente muestran cierto nivel de insatisfacción que es importante tomar en cuenta para efectos de lograr el máximo nivel de satisfacción.

\section{Gráfico 7. Amabilidad de los empleados con los clientes}

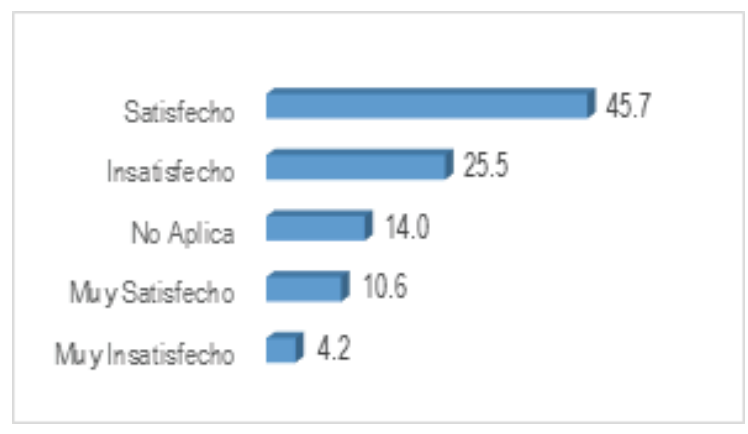

Fuente: Elaboración propia

\section{Gráfico 8. Disposición de los empleados para ayudar a los clientes}

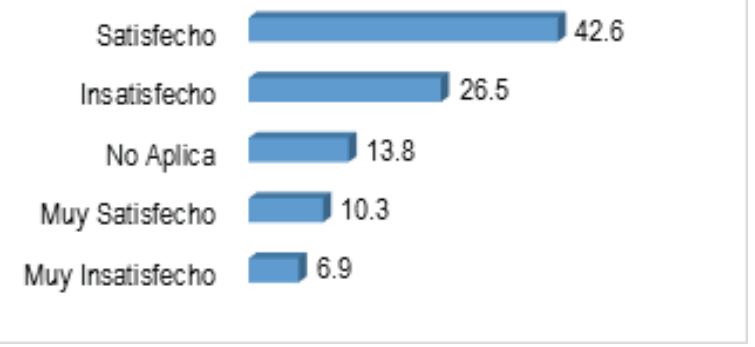

Fuente: Elaboración propia

De igual manera, los entrevistados solicitan mayor variedad de artículos promocionales que sean creativos y de calidad, además que debe haber mayor disponibilidad de artículos o equipos para las diferentes carreras (calculadoras, papelería, dispositivos electrónicos, instrumento logia de las carreras, stickers, CD, DVD, cuadernos, mochilas, folders entre otros).

Las sugerencias que dan los miembros de la comunidad universitaria para que la librería pueda mejorar, se observa en el gráfico 9 , es que realicen más publicidad por 
diferentes medios de comunicación como las redes sociales (Facebook, Instagram, Twitter), tomando en cuenta que el mayor porcentaje de la comunidad universitaria, que son estudiantes y docentes sí hacen uso y están expuestos a estos medios. También se menciona la importancia de que los estantes estén debidamente rotulados para hacer más agradable y fácil la búsqueda de los productos.

\section{Gráfico 9. Recomendaciones para mejorar las ventas en la librería}

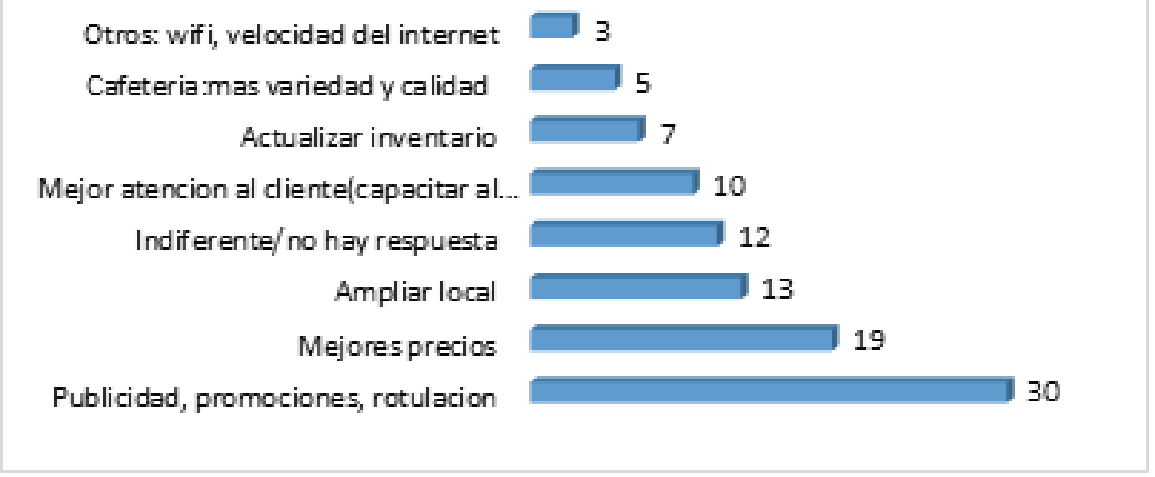

Fuente: Elaboración propia

También recomiendan que ocasionalmente ofrezcan promociones reales donde puedan sentir que, a la administración de la librería, le interesa satisfacer y agradar al cliente.

Es por eso que se puede observar que en la segunda posición, las personas recomiendan bajar precios para poder realizar siempre sus compras en esta librería y no tener que buscar en la competencia por "unos cuantos lempiras menos" en los precios.

Así como crece la comunidad universitaria, crece la necesidad de ofrecer un espacio más amplio en la librería y sobre todo si se quiere manejar el concepto de librería y café que a pesar de ser una excelente idea, el espacio ya no es suficiente y se mezclan diferentes tipos de contaminantes como el ruido y los aromas. 


\section{Cruce de variables}

Tabla 1. Cruce de variables tipo de usuario-amabilidad de los empleados

\begin{tabular}{|c|c|c|c|c|c|c|c|c|c|c|}
\hline \multirow{3}{*}{ Tipo de usuario } & \multicolumn{10}{|c|}{ Los empleados son siempre amables con los clientes } \\
\hline & \multicolumn{2}{|c|}{ No aplica } & \multicolumn{2}{|c|}{$\begin{array}{c}\text { Muy } \\
\text { insatisfech } \\
0\end{array}$} & \multicolumn{2}{|c|}{$\begin{array}{c}\text { Insatisfech } \\
0\end{array}$} & \multicolumn{2}{|c|}{ Satisfecho } & \multicolumn{2}{|c|}{$\begin{array}{c}\text { Muy } \\
\text { satisfecho }\end{array}$} \\
\hline & 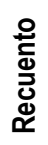 & $\begin{array}{c}\% \text { del } \\
n^{\circ} \text { de } \\
\text { fila }\end{array}$ & 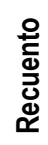 & $\begin{array}{c}\% \text { del } \\
n^{\circ} \text { de } \\
\text { fila }\end{array}$ & 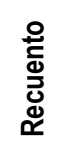 & $\begin{array}{c}\% \text { del } \\
n^{\circ} \text { de } \\
\text { fila }\end{array}$ & 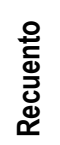 & $\begin{array}{c}\% \text { del } \\
n^{\circ} \text { de } \\
\text { fila }\end{array}$ & 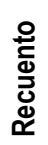 & $\begin{array}{c}\% \text { del } \\
n^{\circ} \text { de } \\
\text { fila }\end{array}$ \\
\hline Estudiante & 46 & 12.2 & 20 & 5.3 & 103 & 27.2 & 184 & 48.7 & 25 & 6.6 \\
\hline Catedrático & 16 & $10 . z$ & 5 & 3.2 & 52 & 33.1 & 66 & 42.0 & 18 & 11.5 \\
\hline $\begin{array}{l}\text { Personal } \\
\text { administrativo }\end{array}$ & 12 & 8.0 & 5 & 3.3 & 25 & 16.7 & 75 & 50.0 & 33 & 22.0 \\
\hline $\begin{array}{l}\text { Personal de } \\
\text { mantenimiento } \\
\text { y servicio }\end{array}$ & 34 & 40.0 & 2 & 2.4 & 16 & 18.8 & 27 & 31.8 & 6 & 7.1 \\
\hline
\end{tabular}

Fuente: Elaboración propia

Si se analiza en la tabla número 1. el nivel de satisfacción por el tipo de usuario de los servicios y productos de la Librería universitaria, se observa que el personal administrativo muestra mayor satisfacción, sin embargo, son los estudiantes y docentes quienes más hacen uso de estos servicios, y estos muestran mayores niveles de satisfacción e insatisfacción. Una de las principales recomendaciones por parte de los usuarios es precisamente que los empleados sean más amables. El personal de mantenimiento y servicio hace poco uso de los servicios de la Librería universitaria.

\section{Tabla 2. Cruce de variables tipo de usuario-variedad de productos}

\begin{tabular}{|c|c|c|c|c|c|c|c|c|}
\hline \multirow[b]{3}{*}{ Tipo de usuario } & \multicolumn{8}{|c|}{ La variedad de los productos responde a sus necesidades } \\
\hline & \multicolumn{2}{|c|}{ No aplica } & \multicolumn{2}{|c|}{ Muy insatisfecho } & \multicolumn{2}{|c|}{ Satisfecho } & \multicolumn{2}{|c|}{ Muy satisfecho } \\
\hline & 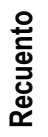 & $\begin{array}{c}\% \text { del } \\
N^{\circ} \text { de } \\
\text { fila }\end{array}$ & 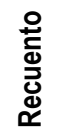 & $\begin{array}{l}\% \text { del } N \text { de } \\
\text { fila }\end{array}$ & 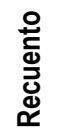 & $\begin{array}{c}\% \text { del } N \text { de } \\
\text { fila }\end{array}$ & 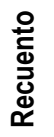 & $\begin{array}{c}\% \text { del } N \text { de } \\
\text { fila }\end{array}$ \\
\hline Estudiante & 40 & 10.6 & 44 & 11.6 & 167 & 44.2 & 24 & 6.3 \\
\hline Catedrático & 22 & 14 & 12 & 7.6 & 65 & 41.4 & 9 & 5.7 \\
\hline $\begin{array}{l}\text { Personal } \\
\text { administrativo }\end{array}$ & 12 & 8 & 6 & 4 & 73 & 48.7 & 17 & 11.3 \\
\hline $\begin{array}{l}\text { Personal de } \\
\text { mantenimiento } \\
\text { y servicio }\end{array}$ & 34 & 40 & 0 & 0 & 31 & 36.5 & 6 & 7.1 \\
\hline
\end{tabular}

Fuente: Elaboración propia 
En relación al nivel de satisfacción sobre los productos ofertados y las necesidades básicas de los usuarios sobre el tipo de producto que busca en este tipo de librería, en general los estudiantes, catedráticos y personal administrativo muestran satisfacción con una tendencia a la insatisfacción reflejada sobre todo por los docentes y estudiantes, según datos de la tabla 2. Un mayor nivel de insatisfacción, aunque no tan elevado como se esperaría, mientras los niveles de insatisfacción son muy relevantes y exigen una acción de mejora.

Tabla 3. Cruce de variables tipo de usuario-disponibilidad de recomendar la Librería Universitaria

\begin{tabular}{|c|c|c|c|c|c|c|c|c|c|c|}
\hline \multirow{3}{*}{ Tipo de usuario } & \multicolumn{10}{|c|}{$\begin{array}{l}\text { En general, estaría dispuesto a recomendar los productos y servicios que ofrece } \\
\text { la librería Universitaria }\end{array}$} \\
\hline & \multicolumn{2}{|c|}{$\begin{array}{l}\text { Definitivamente } \\
\text { Sí }\end{array}$} & \multicolumn{2}{|c|}{$\begin{array}{l}\text { Probable } \\
\text { mente sí }\end{array}$} & \multicolumn{2}{|c|}{ Indiferente } & \multicolumn{2}{|c|}{$\begin{array}{l}\text { Probablemente } \\
\text { no }\end{array}$} & \multicolumn{2}{|c|}{$\begin{array}{l}\text { Definitivamente } \\
\text { no }\end{array}$} \\
\hline & 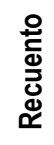 & $\begin{array}{l}\% \text { del } n^{\circ} \\
\text { de fila }\end{array}$ & 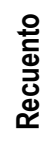 & $\begin{array}{c}\% \text { del } \\
n^{\circ} \text { de } \\
\text { fila }\end{array}$ & 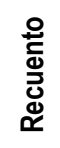 & $\begin{array}{l}\% \text { del } n^{\circ} \\
\text { de fila }\end{array}$ & 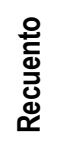 & $\begin{array}{l}\% \text { del } n^{\circ} \\
\text { de fila }\end{array}$ & 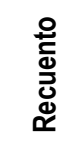 & $\begin{array}{l}\% \text { del } n^{\circ} \\
\text { de fila }\end{array}$ \\
\hline Estudiante & 39 & 10.3 & 148 & 39.2 & 107 & 28.3 & 68 & 18.0 & 16 & 4.2 \\
\hline Catedrático & 23 & 14.6 & 73 & 46.5 & 36 & 22.9 & 24 & 15.3 & 1 & .6 \\
\hline $\begin{array}{l}\text { Personal } \\
\text { administrativo }\end{array}$ & 22 & 14.7 & 67 & 44.7 & 46 & 30.7 & 13 & 8.7 & 2 & 1.3 \\
\hline $\begin{array}{l}\text { Personal de } \\
\text { mantenimiento } \\
\text { y servicio }\end{array}$ & 6 & 7.1 & 36 & 42.4 & 33 & 38.8 & 5 & 5.9 & 5 & 5.9 \\
\hline
\end{tabular}

Fuente: Elaboración propia

Analizando la respuesta de los usuarios de mayor representatividad en Centro Universitario en la tabla 3, observamos que los estudiantes probablemente sí (39.2\%) recomendarían los productos y servicios de la librería, aunque un $28.3 \%$ muestra indiferencia a este tipo de consulta. En el caso de los docentes, el $46.5 \%$ un porcentaje representativo menciono que probablemente sí recomendaría a terceros los productos y servicios de la librería mostrando también indiferencia en su comportamiento de respuesta.

Hallazgos del cuestionario aplicado a colaboradores de la Librería universitaria

Después de realizado el trabajo de campo con los miembros de la comunidad universitaria, y basados en las necesidades básicas y oportunidades de mejoras detectadas en estos resultados cuantitativos, se impartió una capacitación sobre atención al 
cliente y merchandising a los 14 colaboradores de la librería. En la capacitación los colaboradores expusieron algunos puntos importantes por los cuales se pueden ver reflejados estos resultados en el estudio, razón por la cual, se decidió analizar lo que pensaba la contraparte en este estudio, que en este caso son los colaboradores que laboran en la Librería universitaria, obteniendo los siguientes resultados de 10 de los 14 empleados:

La mayor parte tiene contacto con los clientes ya que laboran en el área de atención al cliente $(60 \%)$, y el equipo que utilizan es básico (engrapadoras, calculadoras, probador de billetes) (38\%) así como computadoras (31\%).

Actualmente en la librería no se utiliza un software especial para el manejo de inventarios, siendo una herramienta de mucha importancia para poder agilizar las búsquedas o manejo eficiente de los productos que se ofertan en la librería y manejar control de inventarios. También consideran de importancia que puedan contar con internet para búsquedas específicas sobre textos, autores, disponibilidad, síntesis de libros de texto o literatura.

\section{Gráfico 10. Tipo de software que requiere}

Tipo de software que requiere

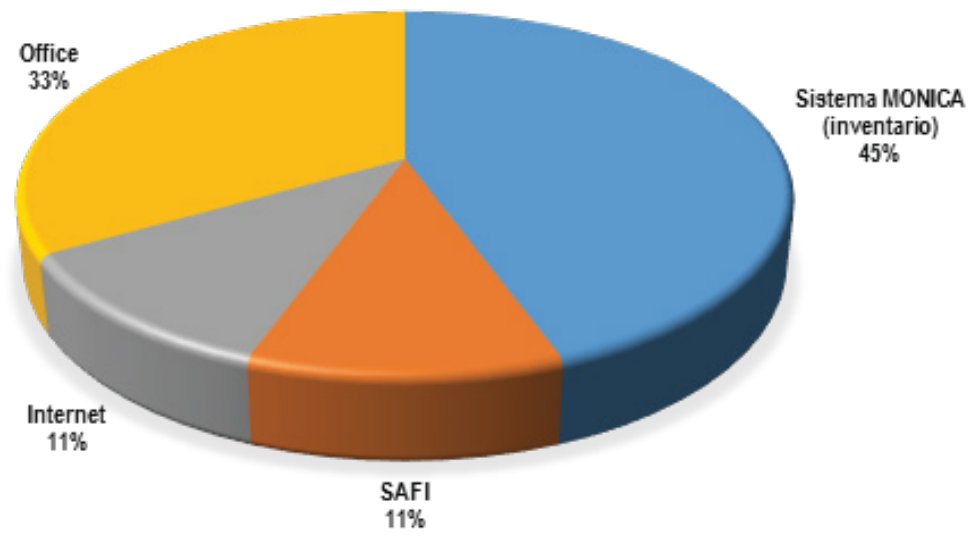

Fuente: Elaboración propia 
Como se puede observar en el gráfico 10, la mayoría de los colaboradores, deben manejar computadoras y software básico como el Sistema Mónica y Office, aunque en su mayoría, los colaboradores expresaron que requieren mayor capacitación con el manejo de paquetes de computación.

La opinión de los colaboradores con respecto a los cambios que estiman convenientes para poder mejorar los procesos en la librería y de esta manera ser más efectivos y eficientes en la atención al cliente, mencionaron que es necesario y conveniente que se pueda trasladar la bodega (15\%) a un lugar más grande y fuera de la librería, pero que pueda estar cerca para poder abastecer con mayor rapidez en el caso que se requiera. Otro punto importante es que se pueda contar con mayor variedad de libros y artículos (10\%) y con mejores precios (10\%). Muchas personas llegan a consultar por variedad de textos con la que no se cuenta y ellos se ven en la situación de decir, no lo tenemos.

Haciendo un recorrido por la librería, es evidente que el espacio físico (10\%) ya es muy pequeño para que se pueda manejar el concepto de librería y café. Al tener mayor espacio físico, su puede ordenar mejor las estanterías por disciplina y temática, colocando una debida rotulación que permita generar una mejor experiencia de búsqueda o que incite a la compra del producto.

Consideran que sería estratégico poder tener reuniones ocasionales con docentes, escritores o lectores frecuentes para poder detectar las necesidades en cuanto a libros de texto o literatura, de esta forma poder hacer las compras necesarias y mantener a los clientes satisfechos.

\section{Gráfico 11. Quejas frecuentes por parte de los clientes}

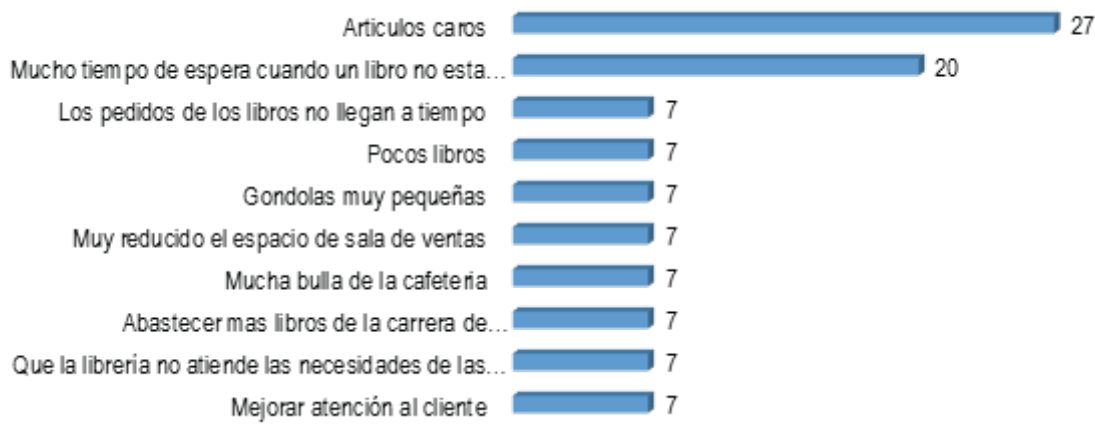

Fuente: Elaboración propia 
Algunas de las quejas más frecuentes que hacen los clientes a los colaboradores, según se muestran en el gráfico 11 , tienen que ver básicamente con los precios de los artículos (27\%) así como el tiempo (20\%) de espera que deben invertir en el momento que un libro no está en góndola y el colaborador se moviliza hasta la bodega a traerlo, se tardan mucho.

Entre otras quejas está el reducido espacio físico entre góndolas, así como la falta de rotulación en cada una de las áreas disciplinarias.

\section{CONCLUSIONES}

- El nivel de satisfacción de la comunidad universitaria en relación a los productos resultó satisfactorio, durante el estudio se mantuvo una tendencia entre satisfactorio e insatisfactorio, lo que hace existan oportunidades de mejoras en algunas áreas.

- En cuanto a la variedad de productos y servicios prestados por la librería, la percepción de los entrevistados está satisfecha, pero con tendencia a la insatisfacción, existen algunos servicios y productos que la población está demandando lo que conlleva a una oportunidad de mercado considerable que se debería implementar en el futuro para tener una satisfacción plena del mercado objetivo de la librería.

- En relación a la atención al cliente estudiado como variable, se determinó que la percepción es que están satisfechos, pero existe inconformidad sobre todo por parte de los estudiantes quienes expresan que no reciben la atención adecuada, que podría mejorar.

- De igual manera con la investigación exploratoria a través del cliente oculto, se pudo establecer que la Librería Universitaria, tiene una ubicación adecuada, con poco espacio y con falta de decoración. Los productos que se ofertan desde textos, elementos publicitarios, uniformes, útiles de igual manera es limitado e insuficientes y poco atractivo para sus compradores. El personal siempre está presente, sin embargo, no ofrece opciones a los compradores.

- En relación a que la cafetería y librería comparten el mismo espacio, se experimenta contaminación ambiental por el ruido y tráfico de personas. 


\section{AGRADECIMIENTOS}

A la Universidad Nacional Autónoma de Honduras por habernos otorgado la beca para poder realizar la investigación a través de la Dirección de Investigación Científica y Posgrados, a la decanatura, a los estudiantes investigadores, colaboradores, personal técnico y de gestión de la DICYP personal del Depto. de Mercadotecnia.

\section{REFERENCIAS BIBLIOGRÁFICAS}

Fisher, L., \& Espejo, J. (2010). Mercadotecnia. México: McGraw Hill. Grande, I. (2000). Markemnng de los Servicios. España: ESIC. Grande, I. (2000). Markeming de los Servicios. España: ESIC.

Kerin, R. A. (2014). Marketing. México: McGraw Hill.

Kotler , P., \& Keller, K. (2006). Dirección de Marketing. España: ESIC.

Kotler, P. (2009). Dirección de Mercadotecnia. México: Prentice Hall.

Kotler, P. (2014). Fundamentos de Marketing. México: Pearson.

Kotler, P., \& Armstrong, G. (2012). Fundamentos de Marketing. México: Pearson.

Kotler, P., \& Cruz , C. (2010). Marketing 10 edición. México: Prentice Hall.

La Tribuna. (2012). Librerias en Honduras. Recuperado de: http://www.latribuna.hn/2012/02/05/las-librerias-en-honduras-1851-1950/

Lehman, D., \& Winer, R. (2007). Administración del Producto. México: McGraw-Hill Interamericana.

Lehman, D., \& Winer, R. (2007). Administración del Producto. México: McGraw-Hill Interamericana.

Mankiw, G. (2010). Principios de economía. México: MacGraw Hill.

Marketingpower.com. (s.f.). Marketingpower.com. Recuperado de: http://www.marketingpower.com/mg-dictionary.php

Roberto Hernández Sampieri, C. F. (2010). Metodología de la Investigación. México: McGraw Hill.

Simón, A. (2011). Diccionario de Economía. México: Editorial Andrade.

UNAH. (2016). Anuario Estadístico . Obtenido de: https://estadistica.unah.edu.hn/anuario-estadisticol

Walker, O., Boyd H, M., \& Larréché, J. (2005). Marketing Estratégico, enfoque toma de decisiones. Ciudad: México Editorial. McGraw Hill

Walker, O., Boyd H, M., \& Larréché, J. (2005). Marketing Estratégico, enfoque toma de decisiones. Ciudad: México Editorial. McGraw Hill

Wikimedia. (2015). Sistema bibliotecario UNAH. Recuperado de: https://es. wikipedia.org/wiki/Biblioteca_de_la_Universidad_Nacional_Aut\%C3\%B3noma_de_Honduras\#cite _note-2 